\title{
Intraductal Carcinoma of the Parotid Gland Presenting as Parapharyngeal Mass
}

\author{
Yamato Okia Hiromitsu Hatakeyama ${ }^{a}$ Masako Otani ${ }^{b}$

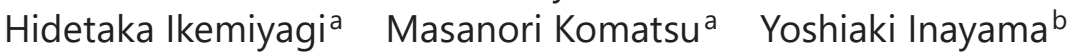 \\ Nobuhiko Oridate ${ }^{c}$
}

a Department of Otolaryngology, Yokohama City University Medical Center, Yokohama, Japan; ${ }^{b}$ Department of Pathology, Yokohama City University Medical Center, Yokohama, Japan; ' Department of Otolaryngology, Yokohama City University Graduate School of Medicine, Yokohama, Japan

\begin{abstract}
What Is It about?
Our case report is about the rare occurrence of a lesion known as intraductal carcinoma in the parapharyngeal space of a 76-year-old male. Intraductal carcinomas are rare malignant tumors originating from salivary gland and the location in the parapharyngeal space is also unusual. We resected through the upper neck approach safely. We diagnosed histopathological intraductal carcinoma with apocrine phenotype. As far as we are aware, this is the first case of intraductal carcinoma from the parapharyngeal space to be reported.
\end{abstract}

\section{Keywords}

Intraductal carcinoma · Parapharyngeal space · Salivary gland tumor

\begin{abstract}
Intraductal carcinomas are rare, malignant tumors that arise from the salivary glands. They commonly grow from the parotid gland and no cases growing from the parapharyngeal space have been reported to date. We report a 76-year-old man who was inadvertently found to have a parapharyngeal lesion by CT scans and MR imaging. The tumor was resected through an upper neck approach and diagnosed histopathologically as intraductal carcinoma. As far as we are aware, this is the first case of intraductal carcinoma arising from the parapharyngeal space. Here, we describe the management of this disease together with a review of the relevant literature.

(C) 2020 The Author(s)

Published by S. Karger AG, Basel
\end{abstract}




\section{Established Facts}

- Intraductal carcinoma of the parotid gland is rare.

- Around 54 cases have been reported worldwide to date.

- Intraductal carcinoma had previously been classified as a low-grade salivary duct carcinoma, but it was classified as a different type of salivary duct carcinoma in 2017.

\section{Novel Insights}

- This is the first case of parapharyngeal intraductal carcinoma to be reported.

- Intraductal carcinoma should be considered in the differential diagnosis of salivary duct carcinoma. In addition to morphology, expression analysis by immunostaining is required for the diagnosis.

- Surgical resection with negative margin offers good prognosis.

\section{Introduction}

Intraductal carcinomas were first described by Delgado et al. [1] in 1996 as "low-grade salivary duct carcinomas." In the past they have been variously referred to as "low-grade salivary duct carcinoma," "low-grade cribriform cystoadenocarcinoma," or "salivary duct carcinoma in situ" $[2,3]$. They were reclassified as intraductal carcinomas by the 4th WHO Classification of Head and Neck Tumors in 2017 [4]. This form of carcinoma is a rare salivary gland tumor that most frequently affects the parotid gland (80.4\%), while being only rarely noted at other sites [2]. No cases growing from the parapharyngeal space have been reported to date. Here, we report a case of intraductal carcinoma in the parapharyngeal space.

\section{Patients and Methods}

\section{Patients}

A 76-year-old man was referred to our hospital with a diagnosis of parapharyngeal tumor that was observed on CT scans obtained for the detection of colon cancer metastasis. On physical examination, we could not palpate the mass from the oral cavity or the outer surface. An MR image revealed a $38 \times 32 \times 34 \mathrm{~mm}$ tumor in the right parapharyngeal space, extending from the bottom of the nasal cavity to the superior dental alveolus. The mass was a tumor lesion with mixed high- and low-signal areas, with the border of the mass being relatively distinct and continuous with the parotid gland. IV contrast-enhanced CT scans contrast revealed the presence of a 3.4-cm fat-containing mass. A radiologist diagnosed the mass as a malignant transformation of a pleomorphic adenoma or other parotid tumor based on the CT scans and MR images (Fig. 1a, b).

\section{Surgery}

All surgical resection was performed within 6 months of the diagnosis. The surgical procedure was performed in the same manner as that for a benign tumor. A 5-cm incision was made in the skin of the upper neck, the skin flap was elevated on the platysma muscle and the 

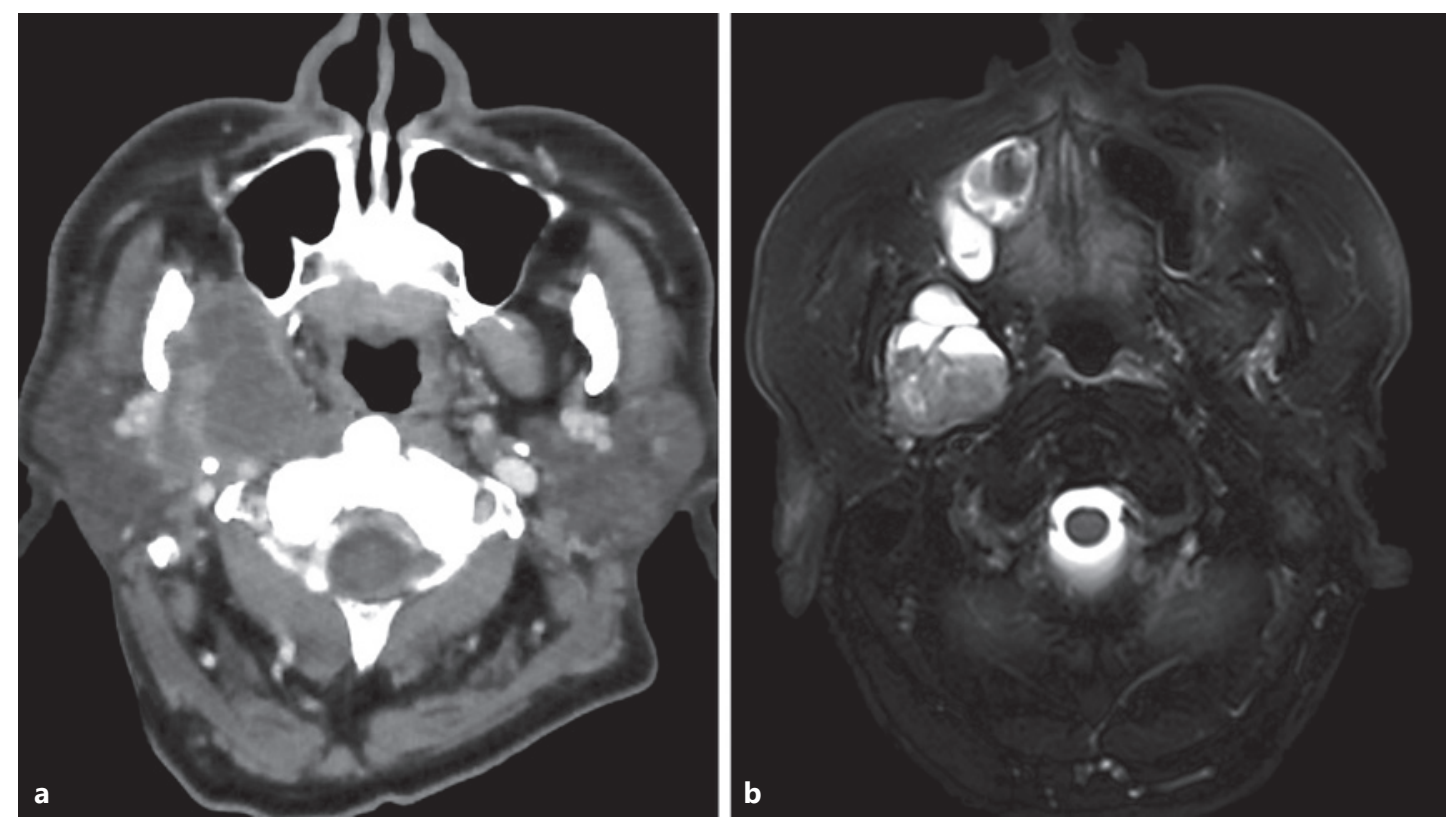

Fig. 1. a An axial IV contrast-enhanced CT scan showing a 3-4 cm fat-containing mass in the right parapharyngeal space. b An axial T2-weighted MR image with fat saturation showing a $38 \times 32 \times 34$ mm tumor containing a mixture of high- and low-signal areas.

marginal branch of the facial nerve was detected and preserved. The tumor was located between the pterygoid muscles and lateral to the epi- and oropharynx. The tumor and surrounding normal parotid tissue was resected en bloc while preserving the facial artery, retromandibular vein, and maxillary nerve. The patient has not experienced any post-surgical complications, and no evidence of recurrence was observed on follow-up CT scans.

\section{Pathological Diagnosis}

The histopathological diagnosis was determined by a pathologist certified by the Japanese Board of Pathology.

The specimen resected from the parapharyngeal space was the tumor as mural nodule within the cystic mass with parotid gland. The tumor was $40 \times 40 \times 32 \mathrm{~mm}$ in size. The cyst contained a white solid tumor with coagulation inside.

Figure $2 \mathrm{a}$ and $\mathrm{b}$ shows hematoxylin-eosin staining of the tumor, which was composed of an irregular cribriform, papillary, or tubular architecture. The tumor cells were columnar in shape and had eosinophilic granules in the cytoplasm and round-to-oval-shaped nuclei with fine chromatin and distinctive nuclei. The mitotic count was $0-1 / 10$ high-power fields. There was a fibrous coat around the cyst with hyalinization, and there was bleeding and inflammatory cells in the capsule. No invasion was seen in the capsule, and the surgical margin was negative. Acini and tubules without atypia were present outside of the capsule, suggestive of salivary gland tissue.

Immunohistochemical staining revealed that mitochondria, androgen receptors, and GCDFP-15 were diffusely positive in the cytoplasm of the tumor cells, whereas HER2 was focally positive. Mammaglobin and S-100 protein were negative for staining (Fig. 2c). The tumor was accompanied with $\alpha$-SMA-, p63-, or calponin-positive myoepithelia (Fig. 2d). The Ki-67 proliferative index was approximately $11 \%$. 

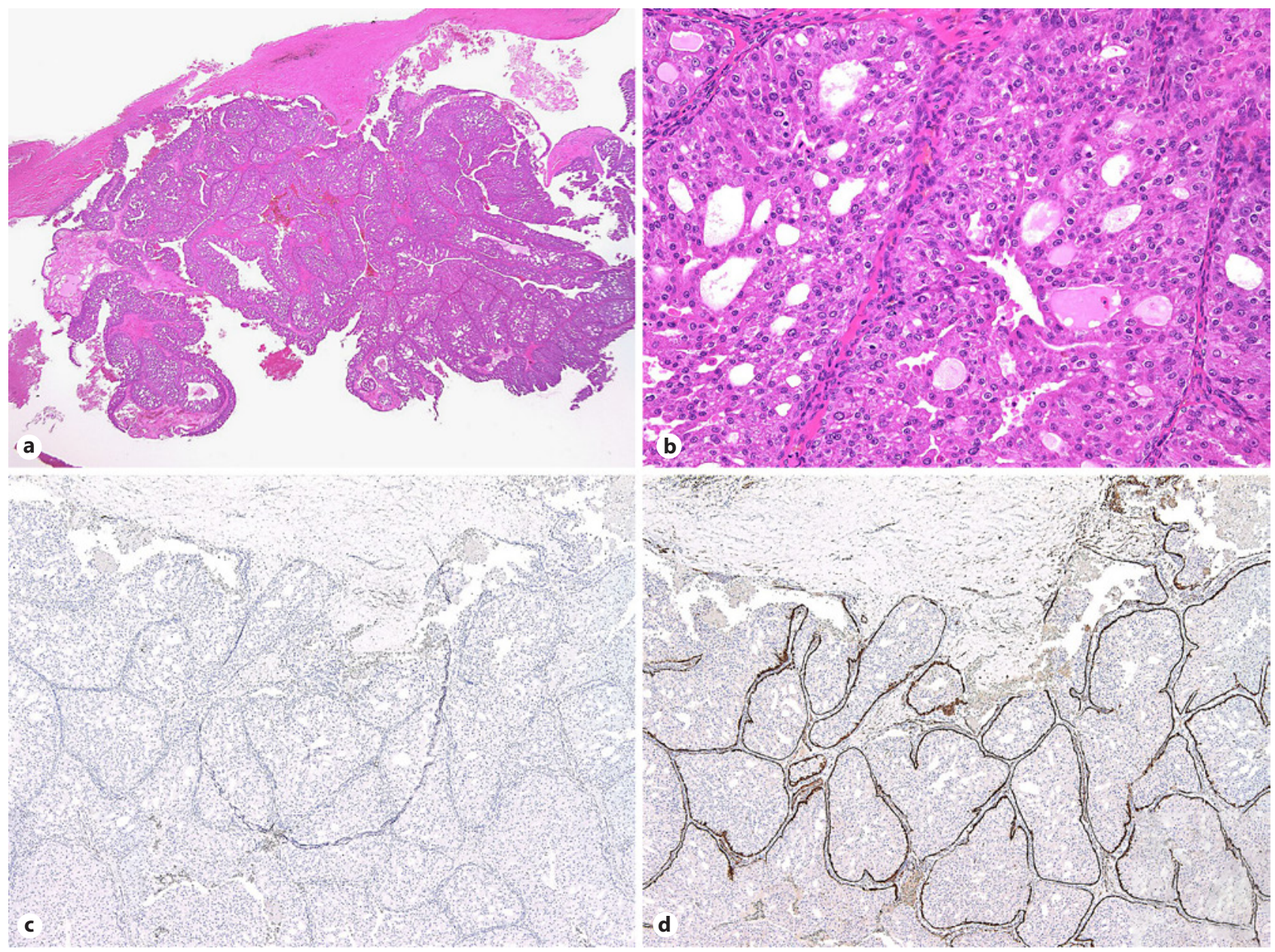

Fig. 2. a HE staining. The tumor is composed of irregular cribriform, papillary, or tubular architecture. b HE staining. The tumor cells are columnar in shape, with eosinophilic granules in the cytoplasm and round-tooval-shaped nuclei with fine chromatin and distinctive nuclei. c Staining for S-100. Cells were negative for S-100 protein. $\mathbf{d}$ Staining for calponin. The tumor is accompanied by calponin-positive myoepithelia.

These histopathological features were morphologically and molecularly consistent with the pathological criteria for intraductal carcinoma of the salivary gland.

\section{Discussion}

The representative histopathological feature of intraductal carcinoma is the intracystic/ intraductal proliferation of neoplastic epithelial cells [4]. Low-grade cribriform cystadenocarcinoma and salivary duct carcinoma in situ were previously categorized as "low-grade salivary duct carcinoma"; however, they have now been collectively categorized as intraductal carcinomas; low-grade and high-grade, respectively $[1,4]$. Intraductal carcinomas are rare salivary gland malignancies that most frequently affect the parotid gland and are only rarely noted at other sites [2]. They are not particularly aggressive and do not commonly invade the surrounding tissues. The tumor mass also grows slowly and facial nerve involvement is rare [2, 4, 5]. Giovacchini et al. [2] summarized 54 cases of intraductal 
carcinoma; however, due to the small number of cases, no statistical significance was observed. Our case is similar to previous cases, in that due to it being painless and asymptomatic, it was only recognized in CT scans obtained for the detection of colon cancer metastasis. A previous report has shown that tumors of the prestyloid space are often salivary gland tumors [6]. This tumor was similarly presumed to be a salivary gland tumor as it was located in the prestyloid space and was continuous with the parotid gland.

\section{Histopathology}

According to the WHO classification, "intraductal carcinoma" is described as typically small, unencapsulated, and cystic. It displays a range of cytological features and can be graded as low-, intermediate-, or high-grade intraductal carcinoma on the basis of the degree of the cytological abnormalities [4]. These carcinomas show a variety of growth patterns, both solid and cystic, ranging from cribriform to solid to micropapillary. Tumor cells are monomorphic and ovoid and are evenly spaced with round nuclei and scant eosinophilic cytoplasm [4]. Additionally, intraductal carcinomas are delimited by a basal/myoepithelial layer, which is p63- and often muscle marker-positive.

The histopathological findings in this case show an irregular cribriform or papillary neoplastic lesion with a cytoplasm showing eosinophilic granules. Further, the tumor cells were covered by myoepithelia that were positive for SMA, p63, and calponin. This case is a purely apocrine variant of IDC because S100 was negative and AR was positive [7].

Thus, we diagnosed intraductal carcinoma with apocrine phenotype. As far as we are aware, this is the first case of intraductal carcinoma from the parapharyngeal space to be reported.

\section{Differential Diagnosis}

Histologically, intraductal carcinoma can mimic other entities in salivary glands including cribriform adenocarcinoma, noninvasive salivary duct carcinoma ex-pleomorphic adenoma (PA), and sclerosing polycystic adenosis (SPA).

The findings of cribriform adenocarcinoma are predominance of cribriform and solid structures with intermingled tubular pattern, mild cellular atypia, 1-3 small inconspicuous nucleoli, and cytoplasm from clear to eosinophilic [8]. Although similar in the points, it is invasive cancer. Intraductal carcinoma is characterized by not having invasive cancer, cribriform adenocarcinoma is negative.

Noninvasive salivary duct carcinoma ex-PA is carcinoma derived from PA and carcinoma occurring within an existing PA [9]. The distinguishing point with noninvasive salivary duct carcinoma ex-PA is no finding of pleomorphic adenoma.

SPA is a reactive, inflammatory lesion of the salivary glands resulting in degenerative fibrocystic changes and adenosis [10]. It frequently harbors intraductal epithelial proliferations with low-grade atypia [10], but the predominant cells are acinar and apocrine without atypia [11]. The findings of this patient were atypical without adenomas and a neoplastic lesion, so SPA is negative.

\section{Treatment}

With regards to treatment, previous reports recommended surgical excision with a negative margin [2]. Lymph node and distant metastases have not been reported in the past and the prognosis is very good after complete excision [2, 4]. Only one case was reported to show recurrence, with Delgado et al. [1] reporting a case of recurrence at 3 months postsurgery in a patient with positive surgical margin. None of the other 53 cases reported showed recurrence or distant metastasis after surgical resection [2]. Giovacchini et al. [2] reported three important points with regard to treatment: (1) surgical excision with a negative margin,

\section{Karger'}


(2) radiation therapy or chemotherapy is unnecessary if imaging results fail to show any evidence of lymph node or distant metastases, and (3) surgical treatment with neck dissection should be performed if lymph node metastasis is suspected [2]. Chemotherapy has been reported for past cases of suspected other cancer types [5], but no regimen has been generally accepted in terms of therapeutic effect for intraductal carcinoma.

The most commonly used diagnostic tool and gold standard has been FNAB; however, it is very difficult to make a differential diagnosis based on cytologic findings of the salivary gland [12]. According to the literature, FNAB does not allow differentiation of this kind of tumor, despite the fact that there is evidence that malignancies were identified in a few cases $[2,5,12,13]$.

This case was a parapharyngeal tumor and we could not perform FNAB or make a diagnosis prior to surgery. We selected surgical resection to allow diagnosis and treatment, and this case has progressed without complications or recurrence.

Based on the excellent outcome, the differentiation of intraductal carcinoma from other salivary gland tumors appears to be important for appropriate treatment decisions and to maximize the quality of life of the patient [2].

\section{Conclusion}

We report a case of intraductal carcinoma in the parapharyngeal space. As far as we are aware, this is the first case of parapharyngeal intraductal carcinoma to be reported. We recommend surgical excision with a negative margin as there is a very positive prognosis after complete excision.

\section{Statement of Ethics}

This is a case report and literature review. Our IRB does not require IRB approval for case reports, although written consent for publication (including images) was obtained from the patient. This research was conducted ethically in accordance with the World Medical Association Declaration of Helsinki.

\section{Conflict of Interest Statement}

The authors declare that they have no conflict of interest.

\section{Funding Sources}

H.H. was supported by a Grant-in-Aid for Scientific Research from the Ministry of Education, Science and Culture. This work was supported by JSPS KAKENHI Grant Number JP 18K09351.

\section{Author Contributions}

Y.O. and H.H. analyzed the MRI. M.O. and Y.I. performed the histological examination, Y.O. was major contributor in writing the manuscript. M.K. and N.O. treated the patients and supplied clinical data. All authors read and approved the final manuscript. 


\section{References}

1 Delgado R, Klimstra D, Albores-Saavedra J. Low grade salivary duct carcinoma. A distinctive variant with a low grade histology and a predominant intraductal growth pattern. Cancer. 1996;78:958-67.

2 Giovacchini F, Bensi C, Belli S, Laurenti ME, Mandarano M, Paradiso D, et al. Low-grade intraductal carcinoma of salivary glands: A systematic review of this rare entity. J Oral Biol Craniofac Res. 2019;9:96-110.

3 Seethala RR, Stenman G. Update from the 4th Edition of the World Health Organization Classification of Head and Neck Tumours: Tumors of the Salivary Gland. Head Neck Pathol. 2017 Mar;11(1):55-67.

4 El-Naggar AK, Chan JKC, Grandis JR, Takata T, Slootweg PJ: WHO classification of Head and Neck Tumours. 2015.

5 Weinreb I, Tabanda-Lichauco R, Van Der Kwast T, Perez-Ordoñez B. Low-grade intraductal carcinoma of salivary gland: report of 3 cases with marked apocrine differentiation. Am J Surg Pathol. 2006;30:1014-21.

6 Kuet ML, Kasbekar AV, Masterson L, Jani P. Management of Tumors Ariging From the Parapharyngeal Space: A Systematic Review of 1,293 Cases Reported Over 25 Years. Laryngoscope. 2015 Jun;125:1372-81.

7 Lu H, Graham RP, Seethala R, Chute D. Intraductal Carcinoma of Salivary Glands Harboring TRIM27-RET Fusion with Mixed Low Grade and Apocrine Types. Head Neck Pathol. 2020;14:239-45.

8 Madhura M, Kumar B, Suma S, Sarita Y. Cribriform adenocarcinoma of minor salivary gland: A mimic of polymorphous low-grade adenocarcinoma. J Oral Maxillofac Pathol. 2016 Sep;3:536-9.

9 Kinoshita K, Yasuda S, Nishida M, Hori S, Yamada M, Togo Y. A case of carcinoma ex pleomorphic adenoma in the submandibular region. Japanese J Oral Maxillofac Surg. 2009;55:424-7.

10 Petersson F. Sclerosing Polycystic Adenosis of Salivary Glands: A Review with Some Emphasis on Intraductal Epithelial Proliferations. Head Neck Pathol. 2013;7:97-106.

11 Barizzi J, Merlo E, Schönegg R, Pelloni R, Mazzuchelli L, Fulciniti F. Pure intraductal carcinoma of the parotid gland: cytologic findings on FNA sample. Report of one case. Diagn Cytopathol. 2017;45:1046-9.

12 Jeong JY, Ahn D, Park JY. Fine-needle aspiration cytology of low-grade cribriform cystadenocarcinoma with many psammoma bodies of the salivary gland. Korean J Pathol. 2013;47:481-5.

13 Obokata A, Sakurai S, Hirato J, Sakamoto K, Takekoshi T, Aoki J. Cytologic features of low-grade cribriform cystadenocarcinoma of the submandibular gland: a case report. Acta Cytol. 2013;57:207-12. 